\title{
La responsabilité des juges dans l'attribution et le traitement des dossiers dans les affaires civiles au sein des tribunaux congolais
}

\author{
Par Professeur Sango Mukalay Adalbert*
}

\section{Abstract : Schedule of responsibilities and the assignment of files at civil courts in Democratic Republic of Congo}

The responsibility of judges in their skill and their treatment of files in Congoleses Courts. It is a big issue, because their attitude and behavior, according to the great deal of people.having files in Congolese courts.

In fully awareness, files are attributed in fully violation according to laws. Which determine the conditions and criterions of attribution. He is the first to add a document to a file, the second one is linked to the lateness do ne in the treatment of the so called files by the judges or the magistrates. In spite of the legal delay, there are both in penal documents or in civil ones.

Through lack of good training of judges and magistrates, after their universities studies together with readiness of oblige, this should be one of the main reason of this behavior. Therefore it is necessary to create a school of magistrates where we will teach to judges and magistrates when they are hired (taken in work).

\section{A. Introduction}

Le droit étant, de tout temps, une réalité sociétale incontournable, les citoyens, de tous les Etats, se posent la question fondamentale, de sa nécessité au vu de certains abus commis, de bonne foi ou non, au nom de ce même droit par les acteurs et professionnels du droit.

Et la République Démocratique du Congo étant, selon la Constitution du 18 février 2006 telle que modifiée à ce jour, un Etat de droit, souverain et démocratique. En tant que tel, elle dispose des juridictions tant civiles que pénales ayant pout mission ou responsabilité première de dire le droit et de condamner.

D'où dans l'exercice de leur profession, les juges ne devraient pas craindre de faire l'objet des poursuites civiles ou pénales en raison des décisions qu'ils rendent de bonne foi dans l'exercice de leurs fonctions. Les erreurs qu'ils commettent, qu'il s'agisse d'erreurs quant à la compétence, à la procédure, à l'interprétation de la loi ou encore aux éléments de preuve, devraient faire l'objet soit d'un contrôle judiciaire ou d'un appel. Pour que l'injustice ne reste pas sans remède, lorsque la situation ne peut être redressée par voie judiciaire

* Sango Mukalay Adalbert est professeur à l'université de Lubumbashi, faculté de droit, et Recteur de l'université de Kalemie, en république Démocratique du Congo. E-mail : adasang@yahoo.fr. 
(en cas de délais excessifs imputables au juge par exemple), le justiciable mécontent devrait réclamer réparation en dirigeant son action contre l'Etat et non contre le juge.

En ce qui concerne l'immunité des juges en matière de poursuites civiles ou pénales, on peut constater que la situation varie au sein des Etats de la famille ou tradition romaniste ${ }^{1}$. Dans les pays dont le système judiciaire est influencé par la Common Law, notamment au Canada et à l'Île Maurice, les juges bénéficient d'une totale immunité contre les poursuites en raison des actes dommageables accomplis dans l'exercice de leur fonction. Il y a lieu de préciser toutefois qu'en ce qui concerne le Canada, l'infraction commise à l'occasion de l'exercice d'une fonction judiciaire ne peut être considérée comme étant commise dans l'exercice de ladite fonction. Une telle faute ne saurait, par conséquent, être couverte par l'immunité contre les poursuites du fait des décisions judiciaires puisqu'elle est commise en dehors de la compétence juridictionnelle du magistrat.

Il faut noter que les poursuites pénales contre les juges peuvent être entreprises dans les cas de forfaiture, d'abus de pouvoir, de concussion, de corruption ou de fraude, on ne peut en conclure que l'impartialité des juges soit menacée ${ }^{2}$ par la possibilité de ces poursuites. Dans les Etats où la prise à partie est possible dans des cas semblables, on devrait pouvoir tirer les mêmes conclusions ${ }^{3}$.

Dans un avis consultatif destiné au Comité des ministres du Conseil de l'Europe, le Conseil consultatif des juges européens fait valoir le fait que, par principe, les juges devraient être absolument dégagés de toute responsabilité civile personnelle à l'égard de toute réclamation les visant directement et liée à l'exercice de leur fonction. Le Conseil suggère que, pour les dommages découlant d'un acte accompli de bonne foi par le juge, l'Etat puisse être directement poursuivi. Il suggère également qu'une action récursoire contre le juge soit possible dans les hypothèses où l'inconduite grave de ce dernier a été établie à l'issue d'une procédure pénale ou disciplinaire.

La Charte européenne sur le statut des juges prévoit d'ailleurs la possibilité d'une action récursoire dans certains cas, notamment en cas de négligence grossière et inexcusable ${ }^{4}$, sous réserve cependant que cette action soit autorisée par un organe indépendant comprenant un nombre substantiel de juges.

1 Michel FROMONT, Grands systèmes des Droits étrangers, Paris 2009, p.7.

2 En Albanie, en Hongrie, en Mauritanie, en Moldavie, en Suisse et en République Tchèque, Sénégal, Tchad, la poursuite pénale doit parfois faire l'objet d'une autorisation préalable du Conseil supérieur de la magistrature, du Président de la République ou du Parlement dans d'autres cas.

3 Le Conseil consultatif des juges européens ajoute que l'acte reproché au magistrat doit avoir été volontaire.

4 C'est le cas du Burkina-Faso, du Maroc, de la Mauritanie, de Haïti, etc. 
Lorsque la responsabilité de l'Etat peut être engagée pour le dysfonctionnement de la justice et lorsque celui-ci est imputable aux faits d'un juge, l'action récursoire est toujours possible ${ }^{5}$. Mais il faut mentionner qu'elle est rarement entreprise.

Dans la plupart des pays qui ont en partage l'usage du français, la contribution des juges à l'instauration d'un pouvoir judiciaire indépendant et impartial a été majeure ${ }^{6}$.

Pour le cas de la République Démocratique du Congo, le problème est et demeure irrésolu, et je l'aborde sous deux aspects majeurs :

\section{B. L'absence d'une formation adéquate des juges}

Le Juge étant un Magistrat ${ }^{7}$, il est recruté soit après un doctorat soit après une licence en droit. Il est cependant déplorable qu'il n'existe pas encore en République démocratique du Congo une Ecole Nationale de la Magistrature où les récipiendaires Magistrats subiraient une formation particulière en rapport avec leur profession.

L'absence de cette Ecole, comme l'Ecole Nationale d'Administration, expose les Juges à commettre des fautes graves et à être souvent tenus civilement responsables des préjudices causés aux justiciables dans l'exercice de leur fonction. Et pourtant, les anciennes colonies africaines de France ont institué, à l'instar de celle-ci, leurs propres Ecoles Nationales de la Magistrature.

Les colonies anglaises ont adopté le système britannique qui consiste à recruter dans la Magistrature des Avocats d'une certaine ancienneté (ayant bien évidemment une certaine expérience) lesquels subissent d'ailleurs une formation et une sélection particulières.

L'absence d'une formation adéquate de nos Magistrats congolais handicape gravement le cours de la Justice. L'ignorance est pour beaucoup dans l'iniquité des décisions judiciaires. La création et l'installation effective de l'Ecole Nationale de la Magistrature en République démocratique du Congo permettrait aux futurs Magistrats, à leur engagement, d'acquérir la compétence technique nécessaire qui constitue une garantie non seulement juridique (relatif au respect des textes existants) mais aussi judiciaire (tributaire des décisions judiciaires) pour les justiciables et les éviterait d'être tenus civilement responsables des fautes commises au préjudice des justiciables dans l'exercice de leur fonction.

On ne saurait, en effet, perdre de vue que l'une des garanties judiciaires pour un procès équitable est le droit à un bon juge. Le droit à un bon juge signifie que l'Etat qui institue les

5 Cfr. Le Bénin, la Bulgarie, le Burkina-Faso, l’Égypte, la France, la Guinée, la Hongrie, le Mali, le Maroc, la Mauritanie, le Niger, la République Tchèque, la Roumanie, le Sénégal, le Tchad et le Togo.

6 Voir : Guy CANIVET, Les influences croisées entre les Juridictions nationales et internationales : Eloge de la « bénévolence », AHJUCAF. http://www.ahjucaf.org/, 22/07/2016.

7 Le mot Magistrat vient du latin, « Magistratus », c'est-à-dire, la charge, la fonction publique, la Magistrature. Au sens strict du terme, c'est toute personne appartenant au corps judiciaire et investie à titre professionnel du pouvoir de rendre la justice (alors on parle des magistrats du siège) ou de la requérir au nom de l'Etat (magistrats du Parquet ou magistrats débout) : MATADI NENGA GAMAN$D A$, Droit judiciaire privé, Bruxelles 2006, p.64. 
Cours et Tribunaux met aussi à la disposition des justiciables des Magistrats compétents non seulement sur le plan technique mais aussi sur le plan moral ${ }^{8}$. L'idée de la création d'une telle Ecole ${ }^{9}$ se dessine de plus en plus depuis 1989 en République démocratique du Congo.

Mon souhait le plus ardent est que cette Ecole prenne corps maintenant dans l'architecture judiciaire congolaise pour une bonne administration de la Justice.

\section{Le non-respect des textes et des délais raisonnables dans l'attribution et le traitement des dossiers judiciaires : une part indissociable de l'injustice et des arrières judiciaires au sein des cours et tribunaux congolais}

\section{Le non-respect des textes dans l'attribution des dossiers judiciaires}

En droit congolais, ce sont les Premiers Présidents ou les Présidents des Juridictions qui distribuent les affaires entre les sections ou les chambres et fixent les dates d'audience ${ }^{10}$. Ils veillent à l'expédition régulière des affaires ${ }^{11}$.

Ils veillent également à la bonne marche des procédures et prennent toute disposition utile pour éviter les retards ${ }^{12}$. Ils assurent la discipline envers les Magistrats des Cours et Tribunaux et le personnel qui y est attaché. Cela relève du domaine de l'administration de la justice selon le statut et la loi organique sur les magistrats.

Mais il est regrettable de constater que ce texte ne soit pas respecté par nos Juges, et pourtant à ce texte, s'ajoute une loi-organique $\mathrm{n}^{\circ} 13 / 011-\mathrm{B}$ du 11 avril 2013 portant organisation, fonctionnement et compétences des juridictions de l'ordre judiciaire qui insiste sur le respect de délai de prononcé des jugements (trente jours au civil et dix jours au pénal).

Il est donc important que les Présidents des Tribunaux respectent les textes qui régissent le fonctionnement de leurs juridictions et qu'ils sanctionnent disciplinairement les juges qui n'en obéissent pas. Car en RDC, nous avons de bons textes mais la maladie congénitale est, à vrai dire, la non application des textes existants.

8 MATADI NENGA GAMANDA, Le droit à un procès équitable, Bruxelles 2002, p.33.

9 Voir les idées congolaises autour de la création de l'école nationale de la magistrature dans MATADI NENGA GAMANDA, La question du pouvoir judiciaire en République démocratique du Congo, Bruxelles 2001, pp.425-426.

10 Art. 6 al. 2 et 3 de l'Arrêté d'organisation judiciaire 299/79 portant règlement intérieur des Cours, Tribunaux et Parquets.

11 Art. 8 al.1 de l'Arrêté d'organisation judiciaire 299/79 portant règlement intérieur des Cours, Tribunaux et Parquets.

12 Art. 8 al.1, idem. 


\section{Le non-respect des délais raisonnables dans le traitement des dossiers judiciaires}

L'immensité du Pays et l'effectif insuffisant des Magistrats ${ }^{13}$ ainsi que l'absence du juge mise en état rendent toujours la procédure lente devant les Cours et Tribunaux congolais; et c'est là la source de plusieurs dégâts dans la procédure congolaise. Car ne dit-on pas que « la justice lente est une forme d'injustice? » et Maitre ABIKHZER Franck ${ }^{14} \mathrm{~d}^{\text {'en ajouter : }}$ «le temps est un bien si précieux et le délai raisonnable un fruit gorgé de promesses ».

Et pourtant, en droit congolais, l'article 43 de la loi organique $n^{\circ} 13 / 011-B$ du 11 avril 2013 portant organisation, fonctionnement et compétences des juridictions de l'ordre judiciaire (code d'OFCJ) dispose que la chambre qui prend une cause en délibéré en indique la date du prononcé. Le prononcé intervient au plus tard dans les trente jours en matière civile, commerciale ou sociale et dans les dix jours en matière répressive.

Mais malheureusement, cela n'est pas souvent pratiqué dans nos Cours et Tribunaux, les juges recourent souvent à l'ancienne pratique en matière civile surtout quand le jugement prononcé doit être notifié à la partie succombante et c'est à dater de la signification que court le délai de recours et que l'on peut procéder à l'exécution du jugement.

Une critique pertinente vient de l'Organisation Non Gouvernementale "Association pour l'accès à la justice”, ACAJ en sigle. Cette dernière a pris l'initiative de dénoncer le dysfonctionnent observé au sein de nos Cours et Tribunaux sur le non-respect de la loi sus évoquée quant à l'insuffisance des Juges dans certains Tribunaux et les conséquences qui en découlent, notamment le non-contrôle des détentions préventives dans des maisons pénitentiaires en chambre de conseil, le sort des affaires déjà prises en délibéré n'est pas connu par les justiciables. Ainsi, l'ACAJ a interpellé le Premier Ministre et le Conseil supérieur de la Magistrature d'être principalement responsables de cette situation pour n'avoir pas pris des mesures d'accompagnement de la loi précitée.

Dans cette perspective l'ONG $A C A J$ a exhorté le Gouvernement à allouer au pouvoir judiciaire des moyens financiers et logistiques nécessaires pour le bon fonctionnement de la justice et de la protection des droits fondamentaux des citoyens. Et elle a également invité le Conseil supérieur de la Magistrature à prendre des dispositions utiles pour mettre fin au dysfonctionnement de certains tribunaux.

Le délai raisonnable est à la fois une exigence d'une bonne administration de la justice et un droit qui doit être garanti pour chaque justiciable. Ainsi le non-respect de ce délai à l'égard de ce dernier peut engager la responsabilité civile du juge.

L'expérience démontre également que pendant ce long moment de procédure les juges sont souvent corrompus par les justiciables impatients d'où l'existence de plusieurs cas de récusations et de procédures de prise à partie ${ }^{15}$ entamés par les autres justiciables à l'en-

13 La République Démocratique du Congo, dans ses superficies de $2.345 .858 \mathrm{~km}^{2}$ avec une population estimée à plus de 70.000.000 d'habitants, est un Etat qui regorge moins de 2500 Magistrats.

14 Docteur en droit et Avocat au barreau de Marseille.

15 La prise à partie est une voie de recours extraordinaire ouverte contre un Magistrat pour dol, concussion et déni de justice. En effet, tous les peuples ont une très haute idée de la justice. L'on 
contre de ce genre des juges, respectivement devant les Cours et Tribunaux ainsi que devant la Cour de cassation.

Il en découle de cela que le Magistrat qui déshonore la société, le droit et la justice est poursuivi par la vindicte populaire qui se traduit soit de façon brutale soit de façon ordonnée et systématisée par la loi. La prise à partie, dans notre société moderne est l'une des sanctions organisées qui a pour objet la condamnation aux dommages et intérêts et éventuellement l'annulation de l'œuvre du juge ${ }^{16}$.

Mais en République Démocratique du Congo, la procédure de prise à partie n'est pas aisée; car elle ne peut être introduite que devant la Cour de Cassation, or celle-ci étant basée uniquement dans la capitale (Kinshasa); la population la moins nantie de l'arrière-pays ne pourra pas avoir les moyens financiers d'en exercer. En sus, l'autorisation préalable du Premier président de la Cour de cassation à laquelle est soumise son ouverture rend encore complexe la procédure. En effet, il n'est pas facile qu'un Magistrat sacrifie son collègue, ce qui apparaitrait comme une violation de leur lien de solidarité professionnelle.

Voilà pourquoi, dans un Pays comme celui-ci, il serait souhaitable que l'exercice de cette voie de recours étant l'unique mode de sanctionner le Juge qui est poursuivi civilement ne soit pas soumise à une autorisation. Il faudrait également qu'elle soit de la compétence des Cours d'Appel basées dans des Provinces afin que chaque citoyen soit à mesure d'en exercer en cas de nécessité.

\section{Conclusion}

Il apparaît que le juge occupe, dans tous nos Etats modernes, une place centrale dans leur système juridique et politique respectifs. Cela ne veut certainement pas dire que le juge devient un « acteur » devant intervenir dans l'arène de la médiocrité morale et scientifique. C'est pourquoi, pour le cas de la République démocratique du Congo, la mise en place rapide d'une Ecole nationale de la Magistrature, la constitution et l'attribution d'un budget conséquent en faveur de la magistrature et le rappel à l'ordre du juge pour la mise en état des dossiers judiciaires, en vue de la crédibilisation de nos institutions judiciaires auprès des justiciables.

La crédibilité doit émaner des hommes et des femmes ayant la charge de dire le droit dans nos Pays. Le développement est tributaire aussi des institutions judiciaires efficaces et fiables mises en place, lesquelles institutions ne valent rien si ceux et celles qui les animent n'ont pas la confiance des personnes censées les solliciter. Certes, aucun système judiciaire ne peut fonctionner de manière juste et efficace s'il ne contient pas des garanties intrin-

sait déjà que là où il y a des personnes humaines, il existe de fait des conflits et que par conséquent le droit y est présent: «Ubi societas, ibi jus » disaient les romains. Le Magistrat quel qu'en soit son statut dans la société, représente l'idée suprême du juste et incarne ainsi la vertu. C'est pour cela que le rôle régulateur du droit, dont il est l'un des acteurs dans la société, est symbolisé par une balance.

16 MATADI NENGA GAMANDA, Le droit judiciaire, op.cit., p.534. 
sèques propres à assurer, dans la mesure du possible, le respect des normes éthiques les plus élevées auxquelles sont tenus les Juges.

Leur impartialité, leur professionnalisme et leur intégrité revêtent un caractère absolument essentiel pour mériter la confiance du public. Chaque Magistrat doit être conscient qu'il incarne une valeur de justice, fondement du progrès, du développement et de la démocratie. L'Etat de droit est une construction fragile dont la solidité doit être constamment éprouvée. C'est pourquoi, même dans les sociétés où la démocratie est considérée comme étant la plus achevée, il est impératif que les juges soient vigilants et qu'ils exercent, avec les moyens dont ils disposent et dont la suffisance peut être remise en question dans de nombreux pays, leur fonction des gardiens de l' 'Etat de droit' ${ }^{17}$.

$\mathrm{GOETHE}^{18}$ ne disait-il pas que :

"Celui qui reconnait consciemment ses limites est plus poche de la perfection »?

Nos Magistrats doivent, donc, évaluer chaque jour le degré de leur confiance par rapport à eux-mêmes, c'est-à-dire la conformité de leurs sentiments et de leurs penchants aux exigences des institutions, des pouvoirs politiques ou des groupes structurés de personnes qu'ils servent.

\section{E. BIBLIOGRAPHIE SELECTIVE}

- Constitution du 18 février 2006 telle que modifiée à ce jour.

- Arrêté d'organisation judiciaire 299/79 portant règlement intérieur des Cours, Tribunaux et Parquets.

- Guy CANIVET, Les influences croisées entre les Juridictions nationales et internationales : Eloge de la « bénévolence », AHJUCAF. http://www.ahjucaf.org/, 22/07/2016.

- MATADI NENGA GAMANDA, La question du pouvoir judiciaire en République démocratique du Congo, Bruxelles 2001.

- MATADI NENGA GAMANDA, Droit judiciaire privé, Bruxelles 2006.

- Michel FROMONT, Grands systèmes des Droits étrangers, Paris 2009.

17 Article $1^{\text {er }}$ de la Constitution de la RDC.

18 Poète, Essayiste et Dramaturge allemand dans Poésie et Vérité (1811-1814) publié en 1931. 